\title{
Ectopic Thyroid Tissue in the Adrenal Gland Mimicking a Pheochromocytoma
}

\author{
Shu-Chi Wang, MD, Siu-Wan Hung, MD,2 Chen-Hui Lee, MD,4 Hao-Chung Ho, MD, \\ Yen-Chuan $\mathrm{Ou}, \mathrm{PhD},{ }^{1}$ and Cheng-Kuang Yang, $\mathrm{MD}^{1}$
}

\section{Abstract}

Ectopic thyroid tissue in the adrenal gland (ETTAG) usually presents as a well-circumscribed cystic mass on a CT scan. However, the MRI features of ETTAG are incompletely understood. We report a case of ectopic thyroid tissue in the adrenal gland, which demonstrates findings similar to those of a pheochromocytoma on the MRI.

Keywords: thyroid, thyroid dysgenesis, adrenal gland, magnetic resonance imaging, pheochromocytoma

\section{Introduction and Background}

$\mathbf{E}$ CTOPIC THYROID TISSUE is a rare developmental abnormality. The prevalence of an ectopic thyroid is 1 per $100,000-300,000$ persons in the general population and 1 per 4000-8000 patients with thyroid disease. ${ }^{1}$ Ectopic thyroid tissue usually occurs in the base of tongue, but may also develop in the mediastinum or in the subdiaphragmatic organs. Ectopic thyroid tissue in the adrenal gland (ETTAG) is extremely rare. Few reports have described the MRI findings of ETTAG. Herein, we report a case of ETTAG that mimics the MRI features of a pheochromocytoma.

\section{Presentation of Case}

A 29-year-old woman without medical history of hypertension received abdominal sonography during a health checkup, and a left suprarenal mass was incidentally found. Abdominal CT scan revealed a $4.5-\mathrm{cm}$ well-defined mass over the left suprarenal area, without contrast enhancement (Fig. 1). Endocrine survey for adrenal mass including ACTH, cortisol, serum potassium level, aldosterone, rennin level, and urinary vanillylmandelic acid revealed values that were all within normal limits. The left adrenal tumor showed a lower signal than the left kidney on the T2-weighted MRI but was relatively increased after fat saturation (Fig. 2). There was no signal loss between in-phase and out-phase sequences on the T1-weighted MRI. A pheochromocytoma was suspected based on these imaging findings. This patient underwent laparoscopic transperitoneal left adrenalectomy uneventfully.
Grossly, a cystic component, measuring $3.5 \times 2 \mathrm{~cm}$ in size, was noted in the resected adrenal gland (Fig. 3). Microscopically, the cyst lining cells and follicular cells were positive for thyroglobulin and thyroid transcription factor-1. Thyroid differentiation was confirmed. Postoperative thyroid

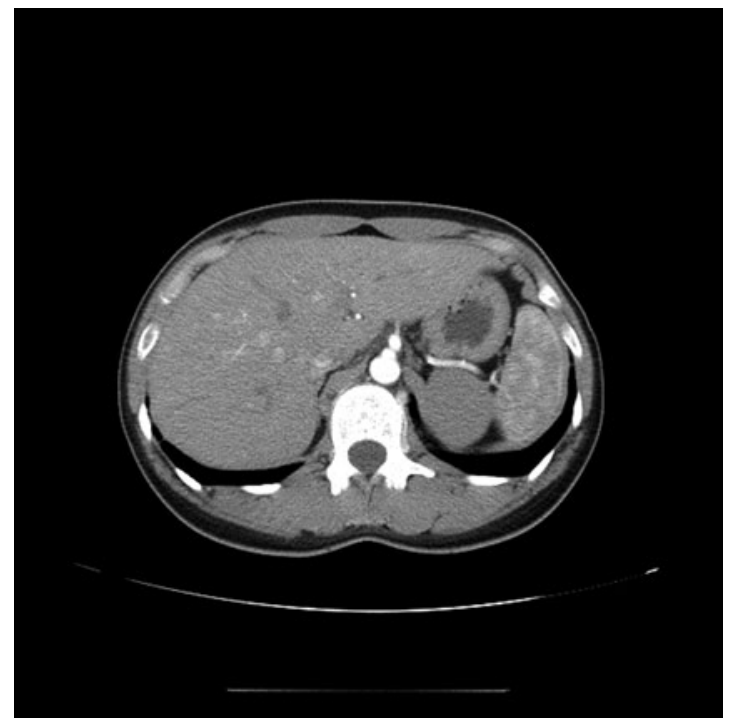

FIG. 1. Abdominal CT scan showing a well-defined mass in the left adrenal gland.

\footnotetext{
${ }^{1}$ Division of Urology, Department of Surgery, Taichung Veterans General Hospital, Taichung, Taiwan, ROC.

Departments of ${ }^{2}$ Radiology and ${ }^{3}$ Pathology, Taichung Veterans General Hospital, Taichung, Taiwan, ROC.

${ }^{4}$ Department of Nutrition and Institute of Biomedical Nutrition, Hungkuang University, Taichung, Taiwan, ROC.
}

(c) Shu-Chi Wang et al. 2017; Published by Mary Ann Liebert, Inc. This is an Open Access article distributed under the terms of the Creative Commons Attribution License, which permits unrestricted use, distribution, and reproduction in any medium, provided the original work is properly cited. 

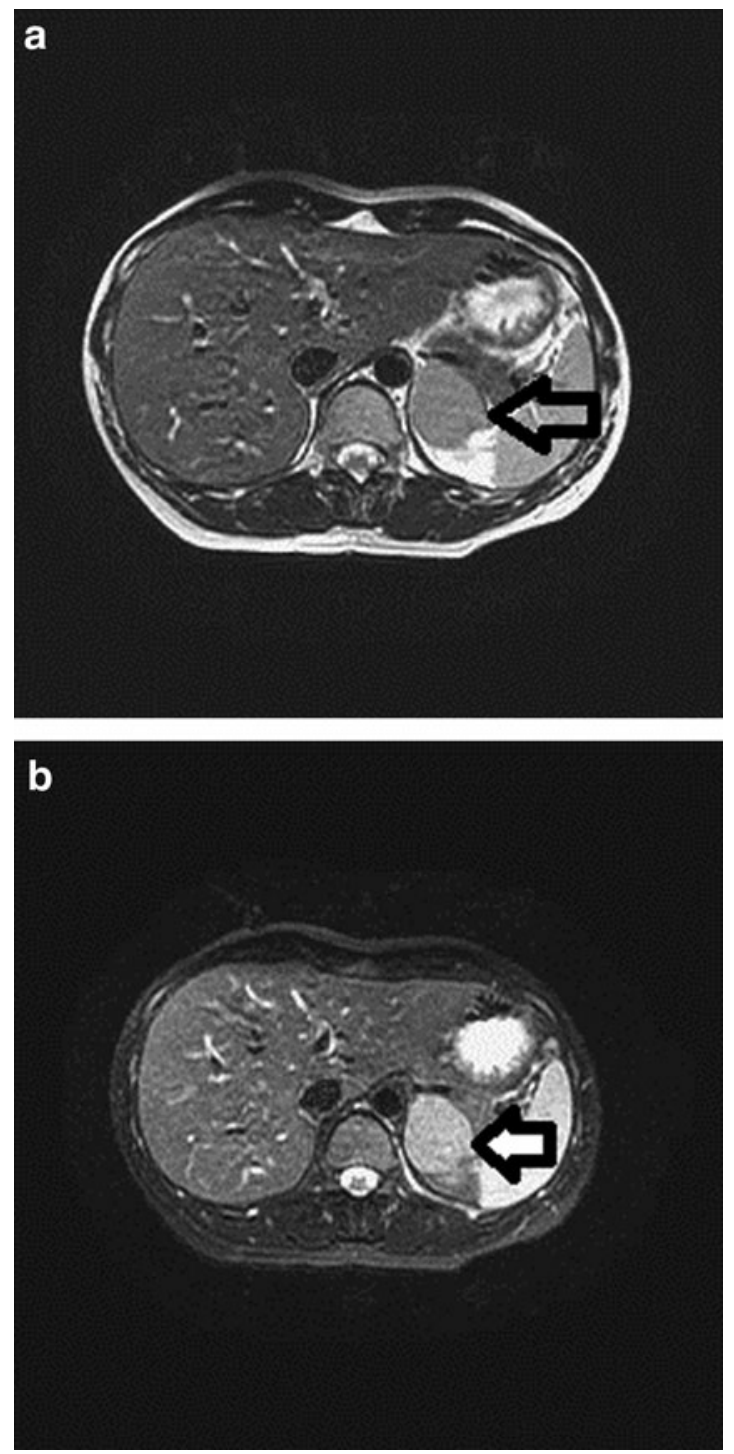

FIG. 2. (a) The left adrenal mass (arrow) shows relatively low signal intensity on axial nonfat-suppressed T2-weighted MRI. (b) The left adrenal mass (arrow) shows increased signal intensity on axial fat-suppressed T2-weighted MRI.

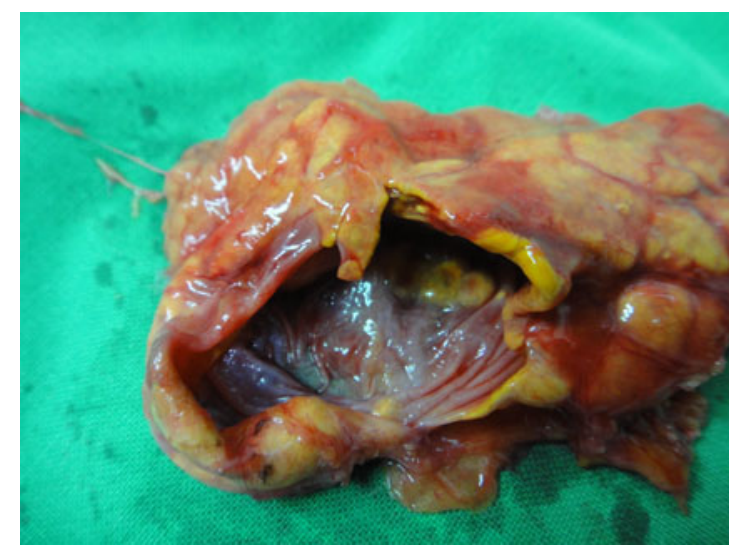

FIG. 3. Macroscopically, the left adrenal tumor contains a cystic component. sonography and chest CT failed to show any lesions in her thyroid gland or disclose any other lesion in the chest.

\section{Discussion and Literature Review}

Ectopic thyroid tissue occurring in the adrenal gland is extremely rare. It is currently unclear how its occurrence could be explained based on embryogenesis. The adrenal gland is located far from the thyroid gland and is outside the primitive thyroid migration pathway. Tsujimura et al. reported the first case of ETTAG in $1996 .{ }^{2}$ Until now, there have been only 11 cases reported in the literature. Most previously reported cases of ETTAG were found in middle-aged women and were unilocular cystic lesions occurring in the adrenal cortex. ${ }^{1}$ Other adrenal tumors, such as adrenal cortical carcinomas, adrenal cortical adenomas, and pheochromocytomas, were also reported to form benign-appearing adrenal cysts. ${ }^{3}$

When the results of a CT scan are indeterminate, MRI or adrenal biopsy may provide more information. MRI is commonly used to differentiate adrenal tumors. However, MRI findings were described in only three of previously reported studies $^{3}$ and the results of the MRI studies of ETTAG did not reveal any consistent findings. In our case, MRI revealed a signal intensity that was slightly lower for left kidney using T2 weighted image but was relatively increased after fat saturation. Moreover, there was no signal loss between in-phase and outphase sequences. These findings were similar to the pattern seen in a pheochromocytoma, which is typically characterized by no signal loss in-phase and out-of-phase sequences. The present case is the first of its kind to demonstrate that ETTAG exhibited findings similar to those of a pheochromocytoma on an MRI scan.

If surgical pathology confirms the presence of ETTAG, it is important to distinguish it from a metastatic lesion from an occult malignant thyroid carcinoma. HBME1, galectin-3, cytokeratin 19, and CITED1 all showed higher expression in papillary thyroid carcinoma and can be used for differential diagnosis. ${ }^{4}$ Also, imaging studies for detecting coexisting thyroid gland tumor and other possible ectopic thyroid carcinoma should be arranged.

\section{Conclusion}

Although the imaging features of ETTAG are not yet fully understood, this disorder usually presents as a cystic lesion. We describe a rare case of ETTAG mimicking a pheochromocytoma on MRI.

\section{Disclosure Statement}

The authors declare that there are no conflicts of interest related to the subject matter or materials discussed in this article.

\section{References}

1. Guerra G, Cinelli M, Mesolella M, et al. Morphological, diagnostic and surgical features of ectopic thyroid gland: A review of literature. Int J Surg 2014;12 Suppl 1:S3-S11.

2. Tsujimura A, Takaha M, Takayama $\mathrm{H}$, et al. Ectopic thyroid tissue in a cystic adrenal mass. Br J Urol 1996;77:605-606.

3. Tada A, Tanaka T, Takamoto A, et al. Ectopic thyroid tissue in the adrenal gland: CT and MRI findings. Diagn Interv Imaging 2016;97:373-375. 
4. Romero-Rojas A, Bella-Cueto MR, Meza-Cabrera IA, et al. Ectopic thyroid tissue in the adrenal gland: A report of two cases with pathogenetic implications. Thyroid 2013;23:1644-1650.

Address correspondence to:

Cheng-Kuang Yang, MD Division of Urology Department of Surgery Taichung Veterans General Hospital No.1650, Taiwan Boulevard, Sect. 4 Taichung 407 Taiwan, ROC

E-mail: yangck@vghtc.gov.tw

\begin{tabular}{|c|}
\hline $\begin{aligned} & \text { Abbreviations Used } \\
& \mathrm{CT}=\text { computed tomography } \\
& \text { ETTAG }=\text { ectopic thyroid tissue in the adrenal gland } \\
& \mathrm{MRI}=\text { magnetic resonance imaging }\end{aligned}$ \\
\hline $\begin{array}{l}\text { Cite this article as: Wang S-C, Hung S-W, Lee C-H, } \\
\text { Ho H-C, Ou Y-C, Yang C-K (2017) Ectopic thyroid } \\
\text { tissue in the adrenal gland mimicking a pheochromocytoma, } \\
\text { Journal of Endourology Case Reports } 3: 1,149-151 \text {, DOI: } \\
10.1089 / \text { cren.2017.0080. }\end{array}$ \\
\hline
\end{tabular}

